\title{
Effects of Erbium on the Properties of Electrochemically-Deposited Zirconium Telluride Thin Films
}

\author{
Imosobomeh L. Ikhioya ${ }^{1}$, Agnes C. Nkele $^{1 *}$, Ezeorba M. Chigozirim ${ }^{1}$, Samson O. Aisida ${ }^{1,2}{ }^{\mathbb{D}}$, Malik \\ Maaza $^{3,4}$, Fabian I. Ezema ${ }^{1,3,4}$ \\ ${ }^{1}$ Department of Physics and Astronomy, University of Nigeria, Nsukka 410001, Nigeria \\ ${ }^{2}$ National Centre for Physics, Quaid-i-Azam University Campus, Islamabad 44000, Pakistan \\ ${ }^{3}$ United Nations Educational, Scientific and Cultural Organization Africa Chair in Nanosciences / Nanotechnology, College of \\ Graduate Studies, University of South Africa, Pretoria, South Africa \\ ${ }^{4}$ Nanosciences African Network (NANOAFNET), iThemba Laboratories-National Research Foundation, Somerset West, Western \\ Cape Province, South Africa \\ E-mail: nechenkele@gmail.com
}

Received: 24 June 2020; Revised: 10 August 2020; Accepted: 13 August 2020

\begin{abstract}
This work studies the effects of varying erbium dopant percentages on the optical, structural, morphological, elemental, and electrical properties of zirconium telluride thin films deposited via electrochemical deposition technique. The obtained films were characterized using Uv-Visible Spectrophotometer, X-ray diffractometer (XRD), scanning electron microscope (SEM), energy dispersive X-ray spectroscope (EDX), and a four-point probe system. XRD analyses showed cubic polycrystalline films with the most intense peak at (111) plane. SEM micrographs revealed spherical balls while EDX spectra confirmed the deposition of basic elements. Good optical features and decreased band gap energies from $1.80 \mathrm{eV}$ to $1.76 \mathrm{eV}$ were observed upon addition of erbium. The electrical conductivities of the films improved significantly upon doping. The deposited films are potential solar cells, and optical materials.
\end{abstract}

Keywords: erbium, zirconium telluride, electrochemical deposition, band gap, solar cell

\section{Introduction}

Thin-film coatings such as antireflective and anti-reflection (AR) coatings are magnified when the thin-film coatings are made up of different layers with quality thicknesses and refractive indices [1]. The market-price of photovoltaic materials used to harvest solar energy such as accumulators can be fabricated into chips of different shapes, sizes, thin metals, and paper using thin-film printing technology. Nanomaterials are also of substantial importance in this fast-growing age $[1,2]$. A common use of thin-film is in the manufacture of reflective and anti-reflective or selfcleaning glasses [3]. In recent years, the study of transition-metal dichalcogenides materials has been extensive because of its applications in photovoltaics $[4,5]$. The transition-metal dichalcogenide materials possess different layers with the transition metal atom sandwiched between two chalcogen atoms [6,7]. Those layers are separated by Van der Waals force $[8,9]$, which offers new promising materials beyond graphene for exploring striking phenomena and device applications [10, 11]. Metallic chalcogenides exhibit excellent optical and electrical features [12, 13]. Researchers have

Copyright (C2020 Agnes C. Nkele, et al

DOI: https://doi.org/10.37256/nat.212021503

This is an open-access article distributed under a CC BY license

(Creative Commons Attribution 4.0 International License)

https://creativecommons.org/licenses/by/4.0/ 
paid much attention to zirconium-based chalcogenides [14,15], transition metal tellurides [16], zirconium telluride [17], effect of temperature on zirconium telluride films [18]. Zirconium telluride $\left(\mathrm{ZrTe}_{2}\right)$ materials have great physical, electronic, thermoelectric, and electrical properties $[17,19]$.

Many researchers have used different techniques to synthesize $\mathrm{ZrTe}_{2}$ thin films; some of which include electrodeposition technique [20], pulsed laser deposition [17, 21, 22], sputtering [23, 24]. Electrochemical deposition is a process that allows oxides or salts to be deposited on the surfaces of conducting substrates by electrolyzing a solution containing the desired metallic ion [25]. It involves immersing the conductive substrate in a solution that produces coatings as electric current passes through the substrate via oxidation and reduction reactions. It serves as a useful synthesis technique because of its quick, efficient, and easy means. Erbium is a rare earth metal that exhibits in a trivalent form $\left(\mathrm{Er}^{3+}\right)$ with sharp absorption spectra bands in the ultraviolet, visible, and near-infrared regions. It finds application in optical amplifiers, optical fibers, metallurgical additive, and laser devices. Amongst these techniques; the electrochemical deposition (ECD) technique is more suitable for the growth / syntheses of erbium-doped zirconium telluride thin film because it is economically friendly, easy to use and can be used for industrial fabrication of films within a short period.

The effect of percentage concentration of erbium on zirconium telluride $\left(\mathrm{Er}^{-} \mathrm{ZrTe}{ }_{2}\right)$ thin films using the ECD technique and its application in solar cell has been studied. Parameters like deposition potential and the percentage concentration of precursors have been optimized at initial stages of deposition. The optical, structural, morphological, elemental, and electrical features of the obtained samples were examined using various techniques alongside the effects of erbium on the properties of zirconium telluride.

\section{Experimental details}

\subsection{Materials and method}

Analytical grade of Zirconium (IV) oxychloride octahydrate $\left(\mathrm{ZrOCl}_{2} .8 \mathrm{H}_{2} \mathrm{O}\right)$, Tellurdioxide $\left(\mathrm{TeO}_{2}\right)$, Hydrogen Chloride ( $\mathrm{HCl})$, Erbium trioxide $\left(\mathrm{Er}_{2} \mathrm{O}_{3}\right)$, deionized water (DW), power supply, multimeter, carbon electrode, fluorinedoped tin oxide as substrate (FTO) were used for the deposition of $\mathrm{ZrTe}_{2}$ and $\mathrm{Er}-\mathrm{ZrTe}{ }_{2}$ thin films. The substrates were dipped in acetone, methanol, rinsed with deionized water, and later ultra-sonicated for $20 \mathrm{~min}$ in methanol solution for purification. The setup of the electrochemical deposition technique (ECD) adopted is shown in Figure 1 with the bath system composed of a cation source (i.e $\mathrm{Er}_{2} \mathrm{O}_{3}, \mathrm{ZrOCl}_{2} .8 \mathrm{H}_{2} \mathrm{O}$ for $\left.\mathrm{Er}^{2+}, \mathrm{Zr}^{2+}\right)$, a source of the anion (i.e Tellurdioxide $\left(\mathrm{TeO}_{2}\right.$ for $\left.\mathrm{Te}^{2-}\right), 100 \mathrm{ml}$ deionized water, magnetic stirrer used to stir the reaction bath, the power supply used to provide an electric field (DC voltage), a conducting glass as the cathode, carbon rod as the anode.

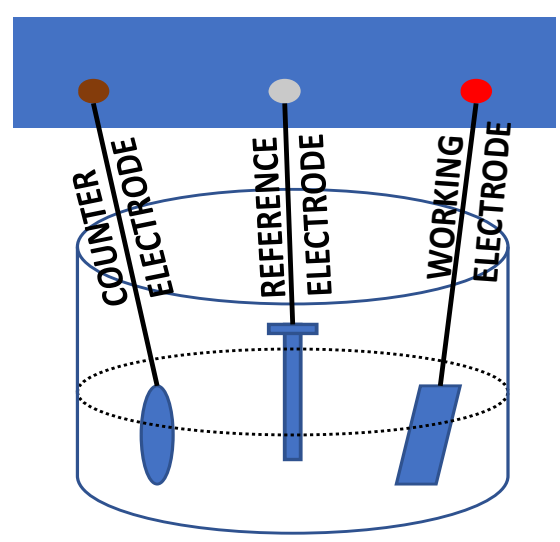

Figure 1. Schematic diagram of a 3-electrode system adopted during ECD process 


\subsection{The growth of Er-ZrTe thin films}

The substrates were kept in a vacuum to avoid contamination. An aqueous solution of 0.1 mol of the cationic precursor $\left(\mathrm{ZrOCl}_{2} .8 \mathrm{H}_{2} \mathrm{O}\right)$ was mixed with $0.15 \mathrm{~mol}$ solution of tellerium powder each in $100 \mathrm{ml}$ of water and stirred. The stoichiometry mixture of zirconium and erbium compounds in $100 \mathrm{ml}$ of deionized water was stirred using the magnetic stirrer at $500 \mathrm{rpm}$ for 3 mins. The FTO substrates were weighed before the deposition. During the deposition of the $\mathrm{ZrTe}_{2}$ film, the target materials were poured into the beakers i.e $15 \mathrm{ml}$ each for the zirconium and tellerium solutions. Using the forceps, the substrate was picked from the glass container, placed in-between the working electrode, and then inserted into the target material. The potential drop across the thin film was measured after 15 sec. with the help of a digital multimeter, a current passing through the sample was measured with the help of a sensitive ammeter while the power supply was kept at a constant voltage of $10 \mathrm{~V}$. Uniform deposition of thin films by ECD was achieved; the deposited films were rinsed in deionized water and kept in an oven to be annealed at $300^{\circ} \mathrm{C}$ for 30 min to remove internal stresses. For the doped samples, $10 \mathrm{ml}$ of the Erbium (Er) solution was added to the above setup for the $1 \%$ doped sample while a similar experimental procedure was adopted for the $2 \%, 3 \%$, and 4\% doped samples. Varied dopant percentages were adopted to obtain varying films of different thicknesses. The substrate-filled films were then weighed after the deposition process to ascertain the film thicknesses. The gravimetric method was adopted in determining the film thickness.

\subsection{Characterizations}

UV-1800 spectrophotometer, Bruker D8 Advance X-ray diffractometer with Cu-K $\alpha$ line $(\lambda=1.54056 \AA)$ in $2 \theta$ range from $10^{\circ}-80^{\circ}$, scanning electron microscope, energy dispersive $\mathrm{X}$-ray spectroscope and a four-point probe was used to measure respectively the optical, structural, morphological, elemental and electrical properties of the deposited films. Various other optical parameters were also calculated and plotted.

\section{Results and discussion}

\subsection{Optical studies}

The optical absorbance and transmittance spectra of the deposited films were studied within a wavelength range of 300-1100 nm as shown in Figure 2a-b. An increase in the absorbance and transmittance was recorded at increasing dopant concentrations of erbium. This shows the positive effect of erbium on $\mathrm{ZrTe}_{2}$ film thereby making it a potential material for solar cells and supercapacitors. Introducing erbium as dopant increased the transmittance of the films with the $4 \%$ erbium-doped sample recording the maximum transmittance. Erbium allowed light passing through it to get absorbed by the host material $\left(\mathrm{ZrTe}_{2}\right)$. Similar absorbance and transmittance results have also been obtained [18].
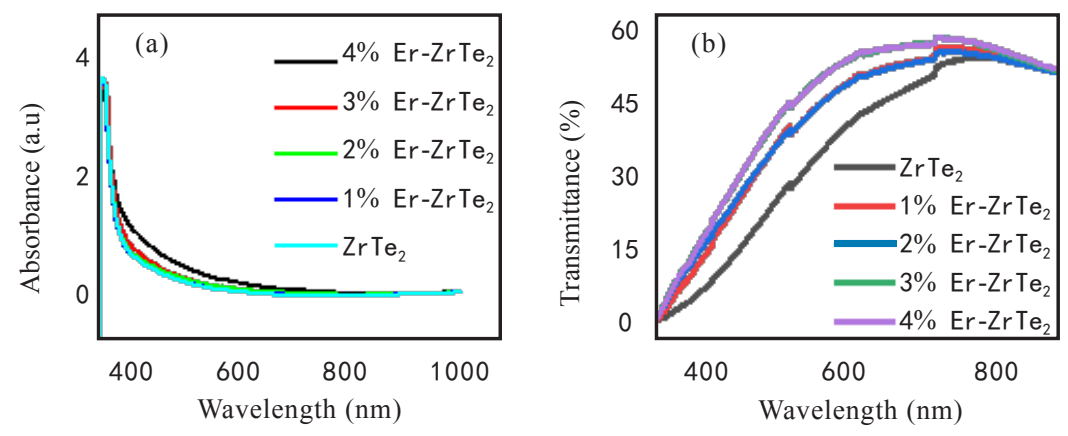

Figure 2. (a) Absorbance and (b) transmittance plots of the deposited films 
The energy band gap spectra of the films shown in Figure 3 estimated from the Tauc plot extrapolation method, using: $(\alpha h v)^{\frac{1}{n}}=\beta\left(h v-E_{g}\right)$. Band gap energies ranging from $1.76 \mathrm{eV}$ to $1.80 \mathrm{eV}$ are outlined in Figure 3 . The band gap energy decreased with increasing dopant percentages. This decrease shows the increased conductivity of the films upon the addition of erbium to the host material. This reduced $\mathrm{E}_{\mathrm{g}}$ also showed high electron movement and that less energy was needed to excite an electron from the valence band to the conduction band [17].

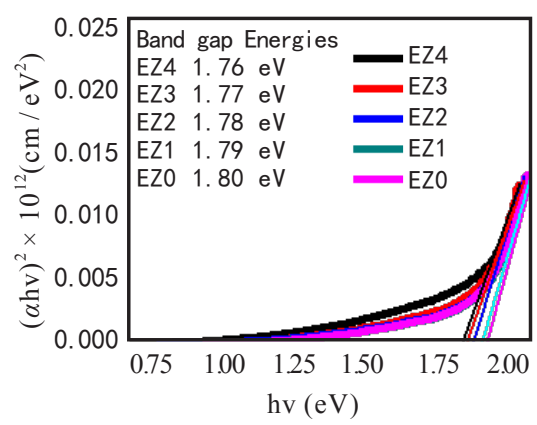

Figure 3. Band gap energy plots of the samples

The optical refractive index, extinction coefficient, and optical conductivity plots are shown in Figure 4a-c. The decreased refractive index values at increasing photon energy showed efficient light propagation through the deposited material. It can be observed that the doped films exhibited better optical properties in terms of conductivity than the undoped sample due to the incorporation of erbium. As the dopant percentage increased, the deposited films exhibited better light absorption and conduction. This feature makes the deposited films potential materials for solar cell fabrication.
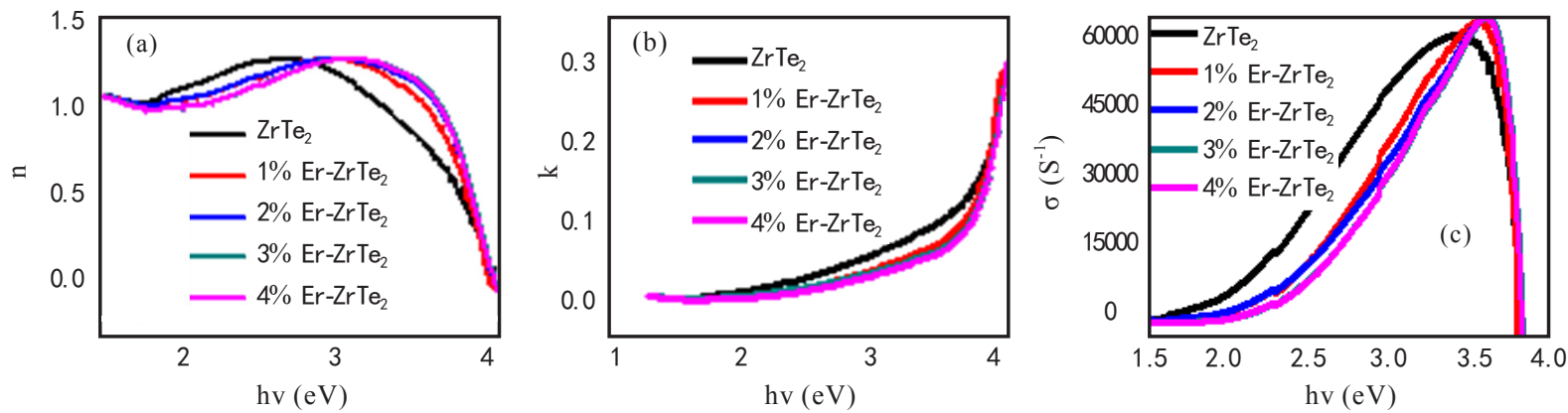

Figure 4. Plots of (a) refractive index (b) Extinction coefficient and (c) optical conductivity versus photon energy for the deposited films

The dielectric constant tells us how strong the electric force is by measuring the amount of electric potential energy in a given material volume. The real and imaginary dielectric constant of $\mathrm{ZrTe}_{2}$ and $\mathrm{Er}-\mathrm{ZrTe} \mathrm{e}_{2}$ thin film as a function of photon energy as shown in Figure 5 reveals that as the dopant concentration of $\mathrm{ZrTe}_{2}$ films increased the photon energy increased. The photon energy of the materials has a steady increase from $2.0 \mathrm{eV}-3.5 \mathrm{eV}$ with a sharp drop in the photon energy of the film up to $4.0 \mathrm{eV}$. This increase signifies effective energy absorption and charge retention. From the plots, real and imaginary dielectric constant values of the films improved due to the introduction of the dopant (erbium). 

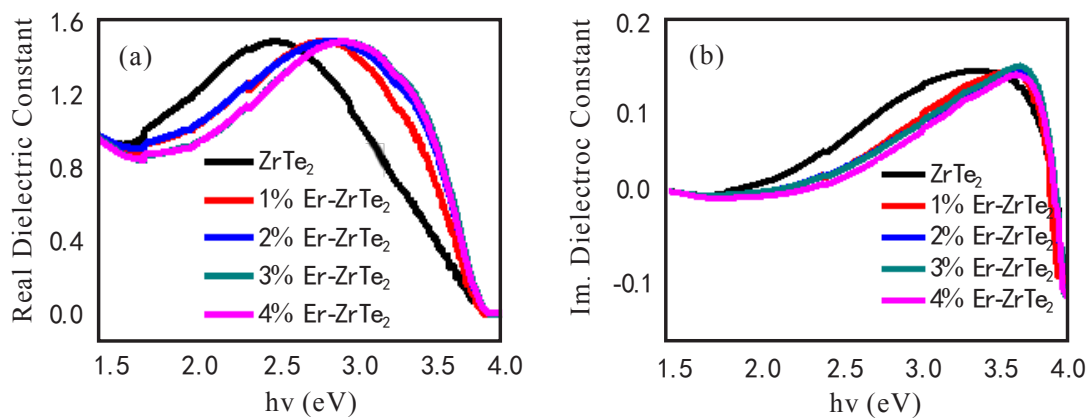

Figure 5. Plots of (a) real dielectric constant and (b) Imaginary dielectric constant against photon energy of the films

\subsection{Structural studies of $Z r T e_{2}$ and Er-ZrTe $e_{2}$ films}

The X-ray diffraction patterns of $\mathrm{ZrTe}_{2}$ and $\mathrm{Er}-\mathrm{ZrTe}_{2}$ thin film showed prominent crystalline peaks that are in agreement with their respective JCPDS card numbers: 046-1088 and 041-1445 for $\mathrm{ZrTe}{ }_{2}$ and Er-ZrTe ${ }_{2}$ films with a cubic phase indexed at (111), (200), (201), and (210) orientations. The XRD patterns as shown in Figure 6 were obtained using the energetic $\mathrm{Cu}-\mathrm{K}_{a 1} \mathrm{X}$-ray source $(\lambda=1.5406 \breve{A})$ in the diffracting angle range of $15-80^{\circ}$. The films were polycrystalline in nature with intense peaks at (111) and (201) planes corresponding to $2 \theta$ values of $26.90^{\circ}$ and $51.42^{\circ}$. Reduction of the peak intensity towards increasing 2 theta degree values is evidence of the presence of microstrain along the crystal lattice. The average crystallite size was estimated using Scherrer's formula in equ. 1. Other structural parameters like lattice constant (a), d-spacing, dislocation density $(\delta)$ have been outlined in Table 1.

$$
D=\frac{k \lambda}{\beta \cos \theta}
$$

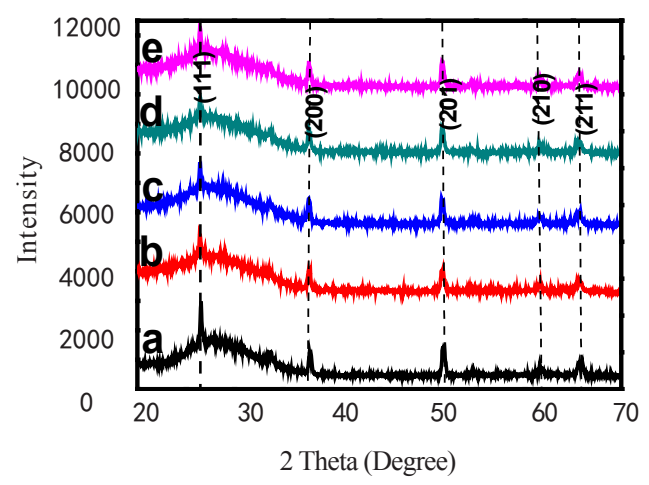

Figure 6. XRD spectra of (a) $\mathrm{ZrTe}_{2}$ and (b-e) 1\%, 2\%, 3\%, 4\% Er-doped $\mathrm{ZrTe}_{2}$ deposited films

Table 1. Structural parameters of $\mathrm{ZrTe}_{2}$ and Er-ZrTe ${ }_{2}$ films

\begin{tabular}{cccccccc}
\hline Sample & $2 \theta\left(^{\circ}\right)$ & $\begin{array}{c}\text { d-spacing } \\
(\mathrm{nm})\end{array}$ & $\mathrm{a}(\mathrm{nm})$ & $\begin{array}{c}\text { FWHM } \\
(\beta)\end{array}$ & $(\mathrm{hkl})$ & $\begin{array}{c}\text { Crystallite Size } \\
\mathrm{D}(\mathrm{nm})\end{array}$ & $\begin{array}{c}\text { Dislocation density, } \\
\delta\left(\operatorname{lines} / \mathrm{m}^{2}\right)\end{array}$ \\
\hline EZO & 26.90 & 0.3310 & 0.3823 & 0.1480 & 111 & 57.622 & 0.00030 \\
EZ1 & 39.52 & 0.2278 & 0.2630 & 0.1851 & 200 & 47.613 & 0.00044 \\
EZ2 & 52.32 & 0.1747 & 0.2017 & 0.2095 & 201 & 44.108 & 0.00051 \\
EZ3 & 62.52 & 0.1484 & 0.1713 & 0.2249 & 210 & 43.833 & 0.00052 \\
EZ4 & 66.13 & 0.1411 & 0.1629 & 0.2258 & 211 & 43.143 & 0.00054 \\
\hline
\end{tabular}


From Table 1, the grain size of the deposited films reduced with increasing dopant percentages due to the formation of impurity bands which allowed the trapping of erbium atoms into the host material. The lattice constant and dislocation density values have also been outlined.

\subsection{Surface morphology of ZrTe ${ }_{2}$ and $\mathrm{Er}-\mathrm{ZrTe} \mathrm{e}_{2}$ thin films}

The surface morphologies of the films are shown in Figure 7. SEM images reveal ball-like crystals of varying sizes that are evenly distributed on the substrate surface. This even distribution confirms uniform surface coverage of the deposited films. Minimal pinholes and cracks were also observed from the SEM images. This compact morphology aided the absorption of the dopant and transmission of light into the deposited films; thereby making it useful for application in solar cells and electronic devices.
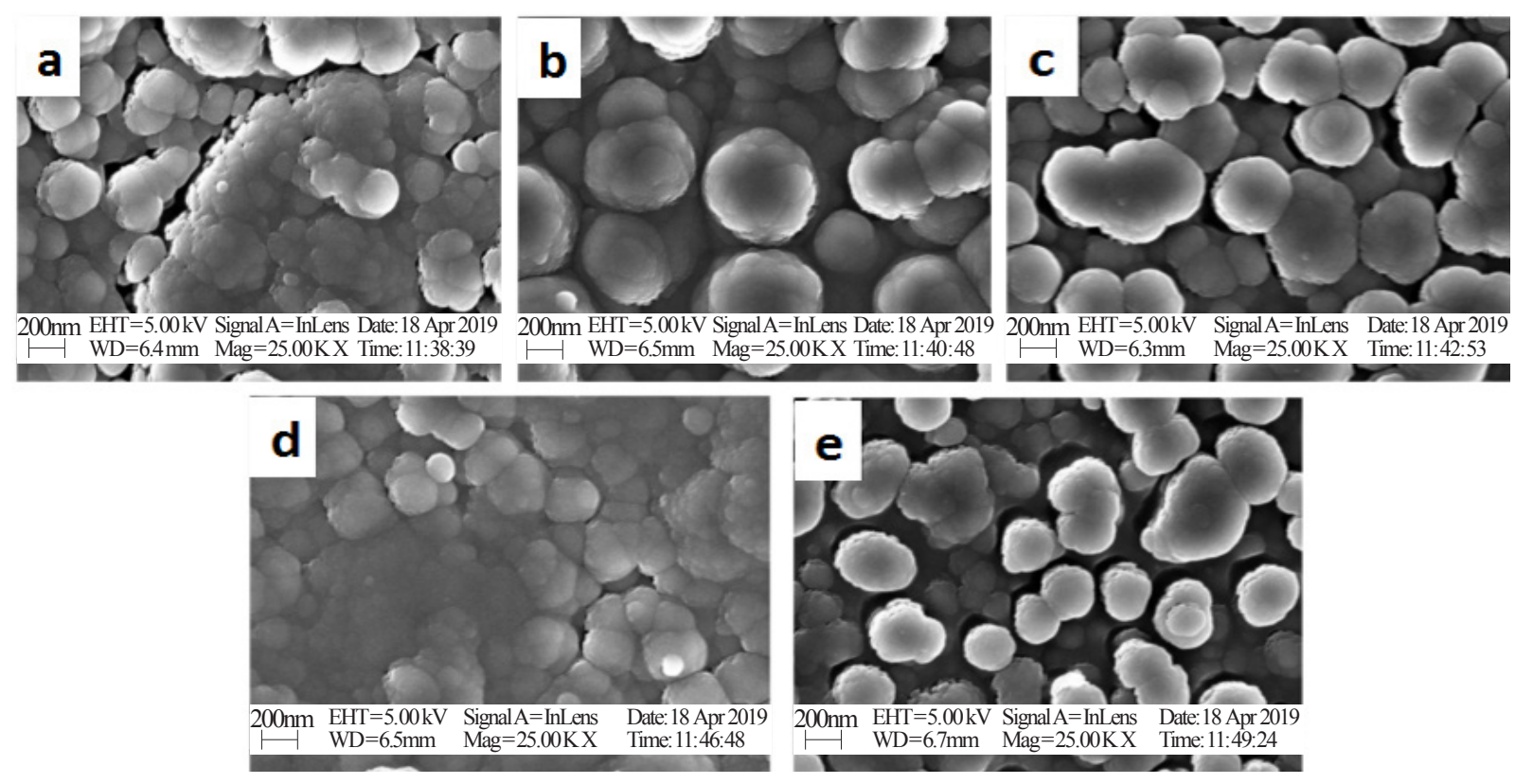

Figure 7. SEM micrographs of the (a) $\mathrm{ZrTe}_{2}$ and (b-e) 1\%, 2\%, 3\%, 4\% Er-doped $\mathrm{ZrTe}_{2}$ deposited films

\subsection{Elemental study of ZrTe ${ }_{2}$ and Er-ZrTe thin films}

The EDX plots of the deposited zirconium telluride and erbium-doped zirconium telluride thin films are shown in Figure 8. The doped samples exhibited similar spectra which are singly shown in the figure. The major elements: zirconium, tellurium, and erbium were observed as constituents of the as-deposited films. These spectra confirmed the deposition of the basic elements. 

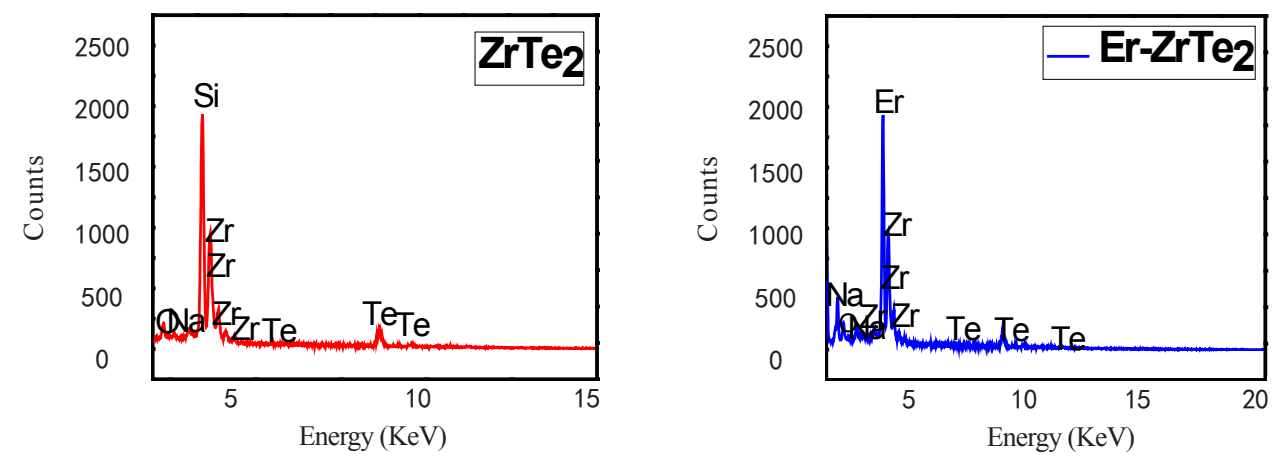

Figure 8. EDX spectra of $\mathrm{ZrTe}_{2}$ and $\mathrm{Er}-\mathrm{ZrTe} \mathrm{F}_{2}$ films

\subsection{Electrical study of $\mathrm{ZrTe}_{2}$ and Er-ZrTe $\mathrm{Z}_{2}$ films}

The electrical parameters of $\mathrm{ZrTe}_{2}$ and $\mathrm{Er}-\mathrm{ZrTe}_{2}$ films are presented in Table 2. Table 2 revealed an increase in the thickness and conductivity of the films at increasing dopant percentages. Introducing the dopant improved the conductivity of the host material (zirconium telluride) and made it potential materials for optical devices. This excellent electrical feature of zirconium telluride films has been reported and make it potential candidates for quantum devices [21].

Table 2. Electrical parameters of $\mathrm{ZrTe}_{2}$ and $\mathrm{Er}-\mathrm{ZrTe} \mathrm{f}_{2}$ films

\begin{tabular}{cccc}
\hline Samples & $\begin{array}{c}\text { Thickness }(\mathrm{t}) \\
(\mathrm{nm})\end{array}$ & $\begin{array}{c}\text { Resistivity; }(\rho) \\
(\Omega \mathrm{m})\end{array}$ & $\begin{array}{c}\text { Conductivity; }(\sigma) \\
\left(\mathrm{Sm}^{-1}\right)\end{array}$ \\
\hline EZO & 130.20 & $6.2973 \times 10^{3}$ & $1.5879 \times 10^{11}$ \\
EZ1 & 140.13 & $5.3303 \times 10^{3}$ & $1.8760 \times 10^{11}$ \\
EZ2 & 144.15 & $4.4021 \times 10^{3}$ & $2.2716 \times 10^{11}$ \\
EZ3 & 149.17 & $3.0041 \times 10^{3}$ & $3.3287 \times 10^{11}$ \\
EZ4 & 152.25 & $6.2973 \times 10^{3}$ & $1.5879 \times 10^{11}$ \\
\hline
\end{tabular}

\section{Conclusions}

Electrochemical deposition was used to grow undoped and erbium-doped Zirconium Telluride thin films. The optical, structural, morphological, elemental, and electrical properties of the deposited films were investigated. The balllike crystalline films exhibited better transmittance with reduced band gap energies upon doping with erbium. Structural studies revealed crystalline films whose crystallite sizes decrease with increasing dopant percentages, elemental studies confirmed the deposition of basic elements while electrical studies confirmed increased conductivity at increasing dopant percentages. Introducing erbium as a dopant improved the properties of the deposited films. Er-doped $\mathrm{ZrTe}_{2}$ thin films find potential applications in solar cell and optical devices.

\section{Acknowledgments and disclosure Acknowledgments}

We acknowledge the sponsorship of TETFUND office (UNN) through the Needs Assessment Intervention Fund. We also thank Nanosciences African Network (NANOAFNET), iThemba LABS National Research Foundation as well as the staff of Nano Research Group, University of Nigeria, Nsukka for their research support. 


\section{Disclosure}

We declare that this manuscript is original, has not been published before, and is not being considered for publication elsewhere. The authors declare no conflict of interest.

\section{References}

[1] X. Shi, et al. Ultrasound wave assisted removal of Ceftriaxone sodium in aqueous media with novel nano composite $\mathrm{g}-\mathrm{C}_{3} \mathrm{~N}_{4} / \mathrm{MWCNT} / \mathrm{Bi}_{2} \mathrm{WO}_{6}$ based on CCD-RSM model. Ultrasonics Sonochemistry. 2020; 68: 104460. Available from: doi: 10.1016/j.ultsonch.2019.01.018.

[2] H. Karimi, et al. An analytical approach to calculate the charge density of biofunctionalized graphene layer enhanced by artificial neural networks. Plasmonics. 2016; 11(1): 95-102. Available from: doi: 10.1007/s11468-0159998-y.

[3] M. Birkholz. Thin film analysis by X-ray scattering. John Wiley \& Sons; 2006.

[4] G. N. Kamm, D. J. Gillespie, A. C. Ehrlich, T. J. Wieting, F. Levy. Fermi surface, effective masses, and Dingle temperatures of $\mathrm{ZrTe}_{5}$ as derived from the Shubnikov-de Haas effect. Physical Review B. 1985; 31(12): 7617.

[5] M. Izumi, T. Nakayama, K. Uchinokura, S. Harada, R. Yoshizaki, E. Matsuura. Shubnikov-de Haas oscillations and Fermi surfaces in transition-metal pentatellurides $\mathrm{ZrTe}_{5}$ and $\mathrm{HfTe}_{5}$. Journal of Physics C: Solid State Physics. 1987; 20(24): 3691.

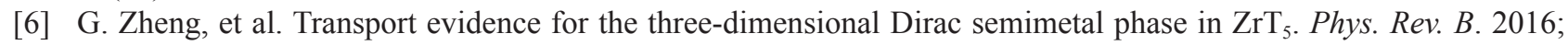
93(11): 1-7. Available from: doi: 10.1103/PhysRevB.93.115414.

[7] G. Manzoni, et al. Evidence for a strong topological insulator phase in ZrTe $_{5}$. Phys. Rev. Lett. 2016; 117(23): 237601. Available from: doi: 10.1103/PhysRevLett.117.237601.

[8] S. Manzeli, D. Ovchinnikov, D. Pasquier, O. V. Yazyev, A. Kis. 2D transition metal dichalcogenides. Nature Reviews Materials. 2017. Available from: https://infoscience.epfl.ch/record/229154 [Accessed 02, May, 2020 ].

[9] Q. Li, et al. Chiral magnetic effect in $\mathrm{ZrTe}_{5}$. Nature Physics. 2016; 12(6): 550-554.

[10] Y. Sun, S.-C. Wu, M. N. Ali, C. Felser, B. Yan. Prediction of Weyl semimetal in orthorhombic MoTe 2. Phys. Rev. B. 2015; 92(16): 1-6. Available from: doi: 10.1103/PhysRevB.92.161107.

[11] Z. Wang, et al. $\mathrm{MoTe}_{2}$ : A type-II Weyl topological metal. Phys. Rev. Lett. 2016; 117(5): 1-5. Available from: doi: 10.1103/PhysRevLett.117.056805.

[12] Y. Zhang, et al. Electronic evidence of temperature-induced Lifshitz transition and topological nature in $\mathrm{ZrTe} 5 . \mathrm{Na}$ ture communications. 2017; 8(1): 1-9.

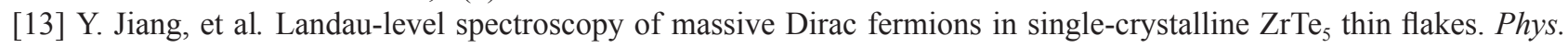
Rev. B. 2017; 96(4): 041101. Available from: doi: 10.1103/PhysRevB.96.041101.

[14] R. Y. Chen, et al. Magnetoinfrared spectroscopy of landau levels and zeeman splitting of three-dimensional massless dirac fermions in $\mathrm{ZrTe}_{5}$. Phys. Rev. Lett. 2015; 115(17): 176404. Available from: doi: 10.1103/PhysRevLett.115.176404.

[15] Z. Fan, Q.-F. Liang, Y. B. Chen, S.-H. Yao, J. Zhou. Transition between strong and weak topological insulator in

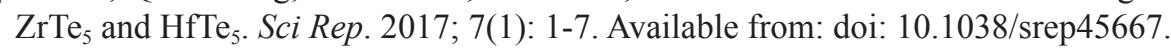

[16] C. H. Chan. Growth and characterization of transition metal telluride thin films. The Hong Kong Polytechnic University; 2019.

[17] H. Wang, C. H. Chan, C. H. Suen, S. P. Lau, J.-Y. Dai. Magnetotransport properties of layered topological material $\mathrm{ZrTe}_{2}$ thin film. ACS Nano. 2019; 13(5): 6008-6016. Available from: doi: 10.1021/acsnano.9b02196.

[18] I. I. Lucky, E. M. Chigozirim, O. Doris O, A. C. Rita, O. C. Ogonnaya. The influence of precursor temperature on the properties of erbium-doped zirconium telluride thin film material via electrochemical deposition. SSRG-IJAP. 2020; 7(1): 102-109. Available from: doi: 10.14445/23500301/IJAP-V7I1P115.

[19] Y. Liu, et al. Zeeman splitting and dynamical mass generation in Dirac semimetal $\mathrm{ZrTe}_{5}$. Nature Communications. 2016; 7(1): 1-9. Available from: doi: 10.1038/ncomms12516.

[20] I. L. Ikhioya, A. C. Nkele, S. N. Ezema, M. Maaza, F. Ezema. A study on the effects of varying concentrations on the properties of ytterbium-doped cobalt selenide thin films. Optical Materials. 2020; 101: 109731.

[21] W. L. Zhu, et al. Magnetotransport properties of the new-type topological semimetal ZrTe. 2017. Available from: http://arxiv.org/abs/1707.00942 [Accessed 02, May, 2020].

[22] P. Tsipas, et al. Massless dirac fermions in $\mathrm{ZrTe}_{2}$ semimetal grown on inAs(111) by van der waals epitaxy. ACS 
Nano. 2018; 12(2): 1696-1703. Available from: doi: 10.1021/acsnano.7b08350.

[23] X. B. Li, et al. Experimental observation of topological edge states at the surface step edge of the topological insulator $\mathrm{ZrTe}_{5}$. Physical review letters. 2016; 116(17): 176803.

[24] R. Wu, et al. Evidence for topological edge states in a large energy gap near the step edges on the surface of $\mathrm{ZrTe}_{5}$. Physical Review X. 2016; 6(2): 1-8.

[25] Y. Zhang, et al. Electrochemical deposition of nickel nanoparticles on reduced graphene oxide film for nonenzymatic glucose sensing. Electroanalysis. 2013; 25(4): 959-966. 